\title{
Haptic Ankle Platform for Interactive Walking in Virtual Reality
}

\author{
Ata Otaran and Ildar Farkhatdinov
}

\begin{abstract}
This paper presents an impedance type ankle haptic interface for providing users with an immersive navigation experience in virtual reality (VR). The ankle platform, actuated by an electric motor with feedback control, enables the use of foot-tapping gestures to create a walking experience like a real one and to haptically render different types of walking terrains. Experimental studies demonstrated that the interface can be easily used to generate virtual walking and is capable of rendering terrains, such as hard and soft surfaces, and multi-layer complex dynamic terrains. The designed system is a seated-type VR locomotion interface, therefore allowing its user to maintain a stable seated posture to comfortably navigate a virtual scene.
\end{abstract}

Index Terms-Locomotion in VR, Walking-in-Place, Human-Robot Interaction, Proprioceptive feedback

\section{INTRODUCTION}

Navigating in virtual reality (VR) environments is important for gaming, training, simulators and other interactive applications. With the ability to move their avatar inside the VR environment, users can discover a whole new world while, in reality, staying inside a small room. Typical ways to control movements of an avatar (or first-person camera) in a VR scene include using joysticks, computer keyboards, pointing arm gestures, pedals, and body motion tracking. The most natural way to implement movements of the avatar is probably to animate it with human-like locomotion kinematics. Human locomotion is a multi-modal activity involving vestibular, somatosensory, proprioceptive, auditory senses and efference copy in addition to vision. However, the VR headset can only provide visual feedback from the avatar's point of view. Limited feedback and sensory conflicts often cause simulator sickness. Studies show that lack of body-centred interaction causes spatial disorientation [1] and reduces immersion [2]. Thus, while joysticks and keyboards are practical for many VR applications (like first-person games, driving simulators); they are not suitable to achieve natural interaction when emulating legged locomotion in VR is required.

Full-body motion tracking can be used efficiently as an interface for locomotion in VR, enabling a user to walk in the physical space and map the measured body kinematics to the virtual space [1], [3]. However, such interfaces require costly motion tracking systems and large physical spaces. The physical limits of a user's real walking environment will limit the application of direct motion mapping during walking in VR. A technique called redirected walking [4] overcomes the limitations of using smaller physical spaces by scaling the mapping for translational and rotational motion in VR. Another method to avoid requirements for large physical spaces is repositioning [5], which is based on using omnidirectional treadmills to physically relocate the

Ata Otaran and Ildar Farkhatdinov are with the School of Electrical Engineering and Computer Science, Queen Mary University of London, London, United Kingdom. Ildar Farkhatdinov is also with the Department of Bioengineering, Imperial College of Science, Technology and Medicine, London, United Kingdom. \{a.otaran, i.farkhatdinov\}@qmul.ac.uk

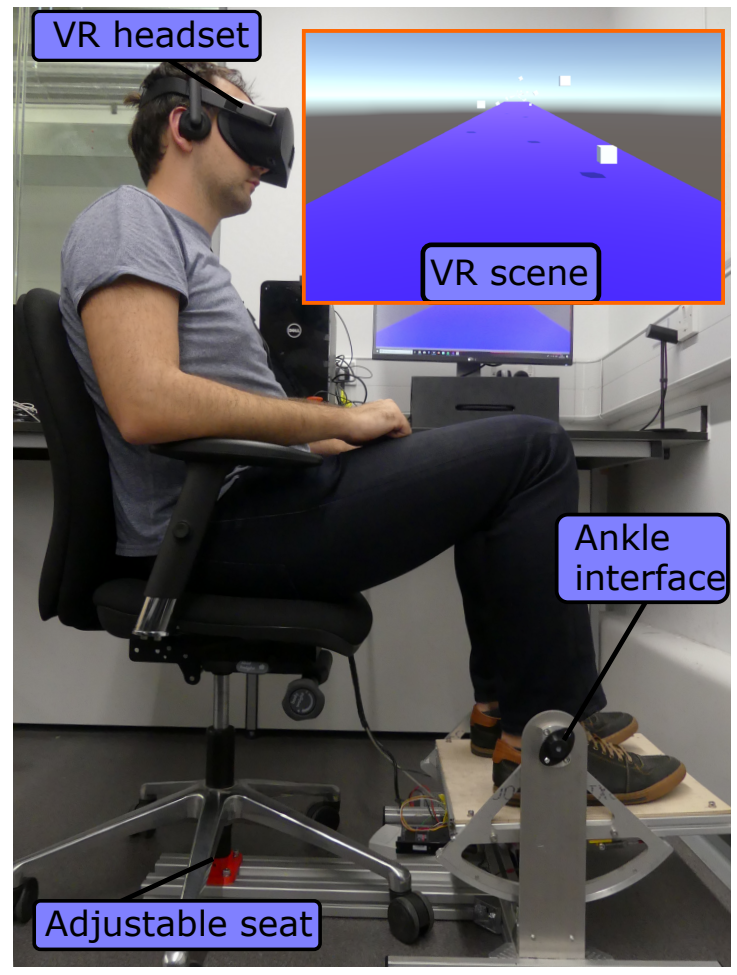

Fig. 1. A user performing foot tapping movements on the platform with visual feedback provided by the VR headset. An explanatory video of the interface is presented in the link: https://youtu.be/wPvGWuyjBI4

user to the initial position. However, such treadmills are complex and contain costly mechatronic systems.

In this paper, we present a walking-in-place type locomotion interface for VR. Our interface, shown in Figure 1 . is based on a user's ankle flexion and requires a small workspace and simple hardware while offering an intuitive walking sensation. Our goal is to demonstrate that the designed interface is suitable for walking in VR, the proposed walking gestures are easy to learn and the interface's actuation capability enables haptic discrimination of different terrains during walking. In contrast to some previous works on VR walking-in-place interfaces [16], [11], [17], we use walking-in-place controlled with foot stepping gestures 
TABLE 1

Comparison of previous works

\begin{tabular}{|c|c|c|c|c|}
\hline Study & Motion Input & Hands-free? & Haptic Feedback & User Posture \\
\hline Slater et. al., 1995 [6] & Marching in place & Yes & None & Standing \\
\hline Feasel et. al., 2008 |7| & Marching in place & Yes & None & Standing \\
\hline Wendt et. al., 2010 & Marching in place & Yes & None & Standing \\
\hline Visell et. al., $2008|9|$ & Stepping & Yes & Terrain feedback through vibration & Standing \\
\hline Son et. al., 2018 [10] & Stepping & Yes & Terrain feedback with MR fluid actuator & Standing \\
\hline Bouguilla et. al., 2003 [11 & Marching in place & Yes & Terrain slope feedback & Standing \\
\hline Nilsson et. al., 2013 [12] & Arm swinging & No & None & Standing \\
\hline Terziman et. al., 2010 [13] & Head movement & Yes & None & Seated \\
\hline Cybershoes [14] & Foot sliding & Yes & None & Seated \\
\hline Templeman et. al., 2007 [15 & Foot sliding & Yes & None & Seated \\
\hline Our interface, 2020 & Ankle movement & Yes & Kinesthetic feedback of terrain & Seated \\
\hline
\end{tabular}

while seated to reduce the fatigue factor and increase body stability. A dedicated foot-ankle robotic platform was developed for implementing the proposed walking-in-place interface. The developed robotic platform enables the control of walking in VR with stepping gestures for a seated user and provides virtual terrain feedback through rendering torques/forces applied to a user's foot/ankle as haptic feedback. The interface proposed in this paper boasts the following advantages: compact and adjustable design to be used by seated users with different body sizes; intuitive and natural walking sensation, achieved through walking gesture-based interaction; ability to provide programmable terrain and slope-related kinesthetic feedback; and simple, low-cost one-degree-of-freedom (1-DoF) actuation and design. The above claims are supported by the experimental study demonstrating that the designed interface was easily used by human participants to perform virtual walking and discriminate different types of terrains. It is important to mention that we propose the use of a 1-DoF ankle platform for walking gestures involving both feet. In contrast to previous research based on separate sub-systems for left and right legs, we demonstrate that a simple 1-DoF robotic ankle platform can be an efficient, intuitive, and natural walking interface for VR applications. To the authors' knowledge, the system presented in this paper is the only seated interface for VR enabling simultaneous natural walking-like input and terrain haptic feedback.

\section{Related REsearch}

This section elaborates on previous attempts to achieve smooth and intuitive mapping of user input to VR locomotion, steering in VR, providing terrain feedback to the user, and having a seated and hands-free interaction. Table 1 summarises pioneering studies that are most comparable to our device in terms of walking-in-place technique and VR environment-related haptic feedback. The last row of the table describes the interface proposed in this paper. We review existing walking in VR interfaces considering their input gestures, type of haptic feedback and whether they can be used in seated postures and allow usage for other manual input devices. As it is demonstrated below our interface has several advantages compared to existing systems. It is based on an ankle actuation platform capable of supporting natural walking gesture input whilst seated and able to provide collocated kinesthetic feedback to render the virtual terrain.

\subsection{Mapping gesture movement data to walking in VR}

The walking-in-place interfaces use periodic body movements to generate a realistic mapping to walking movement in VR. The gestures can be any movement resembling walking, such as marching-in-place, arms swinging, foot sliding, and head movement, as listed in the second column of Table 1 Previous studies used various sensing tools to capture these motion inputs, such as ground force plates [11], [17], a grid of mechanical switch sensors [16], linear encoders on foot sliders [15], external motion capture systems [6], [7], [8], [13], [12], and wearable inertial measurement units (IMUs) [18], [19]. The selection of appropriate sensing technology for walking gesture interfaces is important as it affects the cost, workspace requirements and ergonomics. High-quality motion capture systems require large workspaces and expensive tools [8], [7], [12]; onoff type switches or video camera-based motion capture systems do not provide high-frequency kinematic information [13], [16]; and IMUs required careful adjustment and calibration for each user [18].

Several algorithms for mapping users' gestures into VR walking have been proposed. The algorithms relevant to our study are walking-in-place based methods called Low-Latency, Continuous-Motion Walking-in-Place (LLCM-WIP) [7] and Gait-Understanding-Driven Walking-InPlace (GUD-WIP) [8]. Both of the algorithms use motion capture systems requiring wearable markers on the user's feet and knees (acquired through motion capture systems) to calculate the movements of a VR avatar. LLCM-WIP utilizes the user's low pass filtered heel speed data and maps it to the avatar's forward speed. It offers a very computationally fast and continuous mapping. However, the avatar movement was too swift when the platform angular speed was high. GUD-WIP recognizes certain events in the step cycle and updates avatar velocity with the occurrence of past events. Our algorithm uses the same strategy but requires several different approaches due to the difference in the required motion input and the sensing tool.

\subsection{Terrain feedback during walking in VR}

Only a few interfaces for walking in VR provide haptic terrain feedback (see Table 1] fourth column). Such systems mainly recreated footstep haptic sensation [20], [21], [22] to improve a user's navigation awareness during virtual walking. However, those interfaces used manual input devices to generate virtual walking, and therefore 
haptic feedback and locomotion control inputs were noncollocated. In [10], [23], [24] terrain-based haptic feedback was integrated in shoes. Vibrotactile rendering was employed to display different terrain types to a user during walking in VR. The advantage of such haptic feedback shoe systems is the ability to provide natural interactive walking input and feedback as they were integrated with external motion tracking, however, the limitation is the requirement to have a relatively large physical workspace. Another approach for terrain feedback is to use haptic rendering actuators integrated into the floor [25], [20], [9], but the described floor-integrated feedback methods require additional motion tracking systems and extra workspace for implementing realistic walking inputs. Interestingly, only a few devices for providing kinesthetic feedback for walking in VR have been developed. They are mainly wearable robotic exoskeletons [26], [27] which provide force feedback to the user's lower limbs during walking in VR; however, this feedback is not related to the VR environment but is primarily used for locomotion training. An end-effector type force feedback device that can provide terrain-related feedback was presented in |28]; however, its main disadvantage is the large workspace requirement. Additionally, existing robotic end-effector feedback devices and wearable lower limb exoskeletons are complex in design and control, costly, and therefore, they are less suitable for generic VR applications.

\subsection{Seated, hands-free interaction and steering}

As demonstrated in Table 1 (last column), most of the existing walking in VR interfaces are used while standing and only a few interfaces use seated posture. Marching in place and walking-based interfaces might be more intuitive for locomotion mapping in VR, since walking is a standing activity. However, such standing interfaces require a user to continuously provide extra body muscle effort for maintaining the upright posture which may cause fatigue in the long term. Most standing WIP interfaces require optical motion capture systems to accurately track larger limb movements (merely IMU-based tracking may suffer noise or accumulation of bias in the long term). Even if the walking gesture is confined in a smaller space, optical motion capture systems require a wider occlusion and collisionfree trackable workspace.

VR provides the opportunity to carry out normally standing tasks from a seated posture. From the fatigue and comfort points of view, not having to support one's upright posture throughout the whole VR application, a seated user can conserve more energy compared to a standing user. Therefore, in that sense, seated interfaces are more ergonomic and energy-efficient [29], [30]. Seated interfaces provide a stable upper body posture during locomotion due to the reduced effect of lower body movements on the upper body movements, bodyweight support by a chair, and reduction of the body sways observed in standing [31]. Therefore, seated interfaces are more likely to be suitable for performing fine motor tasks with manual interfaces, such as joysticks, hand-trackers, keyboards, or desktop haptic interfaces.

Avatar steering control is not a major contribution of this paper, however; it is important to take it into account when

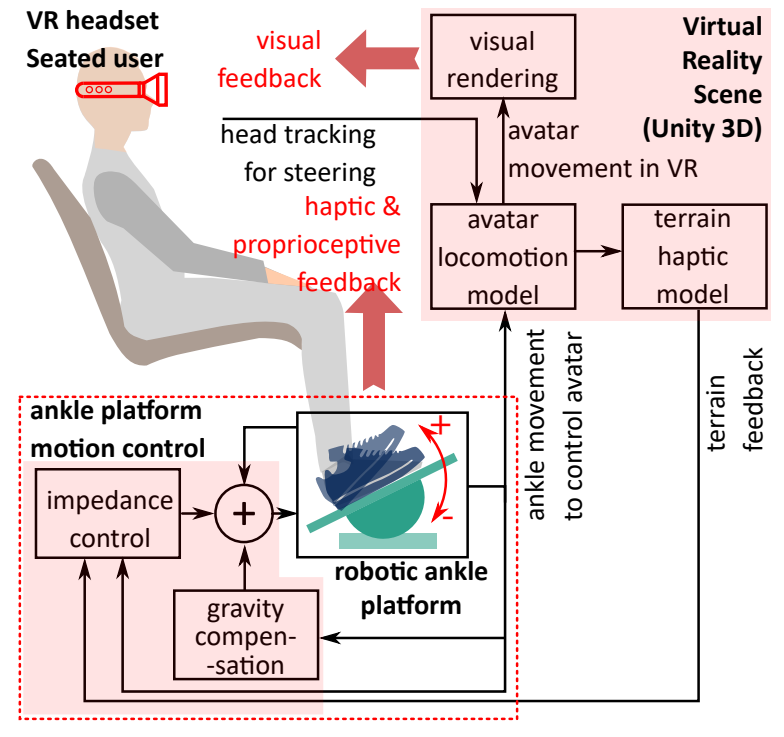

Fig. 2. Block diagram of the motor controller program and the Unity program showing how the physical platform and VR avatar are controlled. Information related to desired avatar speed in VR is transmitted from the motor controller to Unity. Upon making necessary updates on the location of the VR avatar, the corresponding terrain type is transmitted to the motor controller to update related parameters in the impedance controller.

designing seated VR interfaces. Seated interfaces where the user needs to face forward pose a challenge in steering compared to most standing interfaces. Seated interfaces without a large stationary base can use a swivel chair like in [14]. However, seated interfaces that require the user's torso to face forward [15], [13] require additional input cues to control avatar steering. While Templeman et. al. [15] uses joystick buttons, Terziman et. al.[13] uses upper body tilting for steering. In our system, we decided to integrate the approach by Terziman et. al. and test several upper body movement cues to control steering, described in section 4.4

\section{Proposed System}

\subsection{Mechatronic design of the ankle platform}

The proposed system is designed to conform with "general goals for locomotion interfaces" suggested in [32. In particular, the walking (foot-tapping) gesture should be easy to learn and use and should not impose a high cognitive load; the user of the interface should have an ability to use handheld and desktop devices using upper limbs; it should be safe, comfortable, compact, and should not cause fatigue and simulator sickness.

In the following, we present a brief description of the designed robotic ankle interface. The custom-made ankle interface consists of a single 1-DOF actuated platform with a capstan transmission, an electric (DC-) actuator, and control electronics. Figure 1 shows the interface while it is in use, and Figure 2 shows a block diagram of the proposed system, including software and hardware components.

The platform is placed on the floor and if necessary, can be rigidly connected to a chair to improve the stability of the interaction. A seated user places his/her feet on top of the actuated platform such that the ankle flexion/extension is aligned with the platform tilting. The seat location and 

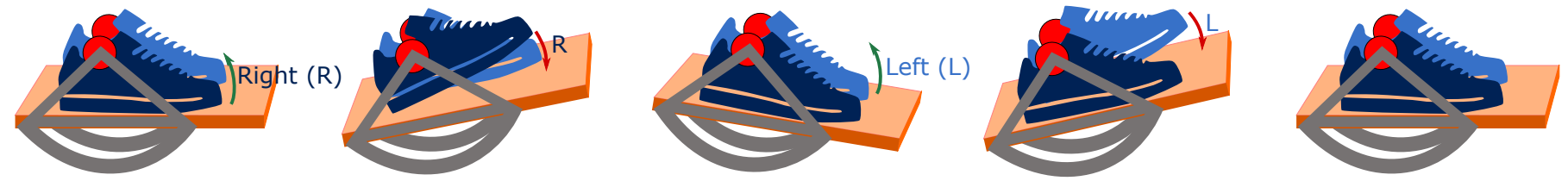

Fig. 3. The preferred walking gestures on the platform where the motion of one foot does not intervene with the motion of the other one.

height can be adjusted and fixed depending on the user's preferred posture and body size by sliding the chair along the rail connected to the platform and adjusting the height of the seat.

The ankle platform is actuated with a geared backdriveable DC-motor equipped with an optical shaft encoder to measure the platform's tilting angle and angular velocity. These measurements were used to detect stepping action (plantar-/dorsi-flexion), and DC-motor control input was used to apply platform torque and generate terrain haptic feedback. In this study, the platform's angle (tilt) was controlled based on the motor shaft encoder measurements and proportional-integral-derivative controller with gravity compensation. The operational workspace of the platform is $\pm 20^{\circ}$. The neutral orientation of the platform $\left(0^{\circ}\right)$ corresponds to horizontal feet; the positive tilt of the platform corresponds to ankle dorsiflexion (foot pointing up), and the negative direction of the platform corresponds to ankle plantarflexion (foot pointing down).

More technical details on the platform and its control can be found in our earlier publication [33], focused on the design, control, and performance assessment of the platform, which offers a more detailed description.

The ankle platform was integrated with a desktop control computer running a custom virtual reality scene implemented in Unity 3D and haptic rendering and ankle control application implemented in C. Oculus Rift CV1 was used as a head-mounted display (HMD).

\subsection{Working principle}

To walk in a VR scene with the help of the designed ankle platform, a user is required to make consequent stepping gestures through left/right ankle plantar-/dorsi- flexion movements, as demonstrated in Figure 3 A normal gait cycle is a quasi-symmetric activity that requires users to perform right and left leg movements with approximately $180^{\circ}$ phase difference. Using seated devices with separate foot interfaces for right and left, it is possible to generate leg movement input that is not dynamically feasible during natural walking due to gravity. However, the use of the single platform for both feet requires a smooth transition between right and left foot movement, similar to how natural walking requires a smooth load transfer between legs. The preferred rhythmic ankle movement is achieved when movements of separate feet do not intervene with each other. For instance, the left foot should only be picked up after the right foot is done pressing down. Although the movement pattern is not practiced by users beforehand, we demonstrate that this can be easily learned and used for interactive walking in VR (section 6.2.

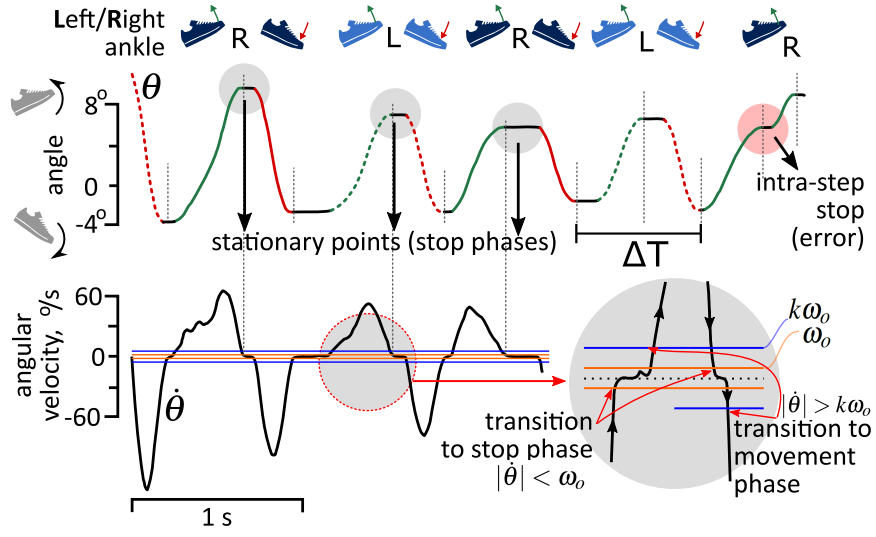

Fig. 4. The platform's angular position and velocity plots recorded during a sample walking test with the proposed ankle flexion gestures. The angular velocity threshold $\left(\omega_{0}\right)$ and classification of different phases are labelled and highlighted with different colours. Stop-walk phase transitions happened when the platform was slow and changing its direction of rotation. Red and green lines on the platform's angular position plot correspond to the right/left ankle's dorsiflexion and plantar flexion. The stop phases where the angular velocity of the platform fell under the threshold $\left(\omega_{0}\right)$ are represented by black regions on the top plot. The intra-step stop is labelled as stopping regions which occur without changing the platform rotation direction. These are correlated with flaws in the right-left rhythmic movement. The time taken between steps, $\Delta T$, refers to the time difference between the most recent stopping event and the one which happened one step earlier.

\section{Algorithm to generate Walking in VR}

\subsection{Imitating real walking with feet gestures}

To develop a natural and easy to learn walking algorithm we first observed a real walking task. Two users, one tall and one average height, were filmed (side view, orthogonal to the sagittal plane) walking on a treadmill at six different velocities, ranging from $2 \mathrm{~km} / \mathrm{h}$ to $7 \mathrm{~km} / \mathrm{h}$. Then, these videos were used to measure and record the associated ankle platform tilting movements. To achieve this, three different users were asked to replicate the walking movements by performing stepping gestures through left/right ankle plantar-/dorsi- flexion movements, as shown in Figure 3. while watching the 12 treadmill walking videos on the screen placed in front of them. Users were specifically asked to replicate the stepping pace instead of matching exact movements. The reason we used videos of two separate walkers is that we were worried that having different styles of walking in the video could affect the movements of the user. However, we did not see any distinguishable change in the platform movements. As a result, we obtained angular displacements for the ankle platform (associated with physical walking) which were then used to design an algorithm for mapping ankle movement to virtual walking.

Following the analysis of the recorded videos and ankle 
platform movements, we needed to determine the corresponding gait phases. It was reasonable to associate the negative peaks of the platform with a foot-tapping movement corresponding to the loading response, and the positive peaks to midstance and terminal stance. Then assuming continuous gait, it is possible to define the anterior-posterior movement of the virtual avatar (or of the first-person viewpoint camera).

\subsection{Walking algorithm}

In our model, we suggest that the speed of the avatar is increasing with the frequency of periodic left and right ankle movements. We propose the following simple kinematic motion model to compute forward walking velocity:

$$
\begin{aligned}
& v=v^{-}+a \Delta t \\
& a=c_{1}\left(\frac{\Delta S}{\Delta T}-v^{-}\right)
\end{aligned}
$$

where $v$ and $v^{-}$are modelled walking velocity at current and previous time instants, respectively; $\Delta t$ is sampling time; $a$ is the walking acceleration, calculated as scaled difference between the commanded, $\frac{\Delta S}{\Delta T}$, and previous velocity, $v^{-}$. The commanded velocity is updated whenever the left or right leg performs a stepping gesture. It is computed based on the time between the two most recent stepping gestures, $\Delta T$ (i.e., the time between the last consequent left and right ankle movements detected based on the platform's encoder). Design parameters, $\Delta S$ and $c_{1}$, correspond to average step length and acceleration scaling factor, respectively.

The kinematic expression (1) computes the modelled velocity, $v$, based on the stepping frequency; however, it does not allow the avatar to quickly stop walking when the user suddenly stops stepping, and therefore, the nonnatural sliding motion of the avatar can occur. To address the above, the model (1) is extended with techniques to detect walking initiation ("walking phase") and stopping actions ("stop phase") based on the angular velocity of a user's ankle performing a stepping action. The user ankle angular velocity, $\dot{\Theta}$, is derived from the ankle platform's shaft encoder measurements. Plots in Figure 4 demonstrate how the transition between the stop and movement phases were defined based on the platform's angular velocity threshold, $\omega_{o}$ and $k \omega_{o}\left(k=3\right.$ and $\omega_{o}=0.4 \mathrm{deg} / \mathrm{s}$, design factors defined empirically based on users' movement patterns). The following rule was used for activating the stop and walking phases:

$$
\begin{gathered}
\dot{\Theta}<\omega_{o} \text { : stop phase, } \\
\dot{\Theta}>k \omega_{o}: \text { walking phase, } \\
\text { otherwise : keep previous phase. }
\end{gathered}
$$

Following this switching rule enabled smooth and continuous virtual avatar velocity, $v_{a}$, calculation:

$$
\begin{aligned}
\text { stop phase: } v_{a} & =v_{a}^{-}+c_{2}\left(-v_{a}^{-}\right), \\
\text {walking phase: } v_{a} & =v_{a}^{-}+c_{2}\left(v-v_{a}^{-}\right)
\end{aligned}
$$

where $v_{a}$ and $v_{a}^{-}$are the calculated VR avatar's walking velocities (body centre of mass velocity) at the current and previous time instant, respectively; $v$ is calculated based
TABLE 2

Key parameters for velocity calculation

\begin{tabular}{l|l|l} 
Parameter & Explanation & Value \\
\hline \hline$\Delta S$ & average step length & $1 \mathrm{~m}$ \\
$\Delta T$ & time taken between steps & calculated \\
$v$ & kinematic model velocity & calculated \\
calculated \\
$v_{a}$ & avatar VR velocity & measured \\
$\Theta$ & angular position of the platform & measured \\
$\dot{\Theta}$ & angular velocity of the platform & $2^{o} / \mathrm{s}$ \\
$\omega_{0}$ & platform angular velocity threshold & 3 \\
$\mathrm{k}$ & multiplier on $\omega_{0}$ setting the threshold & 3 \\
$c_{1}$ & for transitioning to the movement phase & $0.02 / \Delta t$ \\
$c_{2}$ & update coefficient for modeled velocity & 0.1 \\
$\Theta_{D Z}$ & update coefficient for avatar velocity & 0.1 \\
$\Theta_{S T}$ & deadzone thresholds for steering & $\pm 0.05 \mathrm{rad}$ \\
$\Delta t$ & saturation thresholds for steering & $\pm 0.3 \mathrm{rad}$ \\
$\Delta t_{u}$ & motor controller sampling period & $0.002 \mathrm{~s}$ \\
\hline & Unity program fixed update period & $0.016 \mathrm{~s}$ \\
\hline
\end{tabular}

on (1); $c_{2}$ is a scaling design parameter to define the velocity update dynamics. In the stop phase, the avatar will slow down and stop as shown on Figure 5 -b, by the dotted line. In the walking-phase, the VR avatar's velocity, $v_{a}$, will be increased by the computed model velocity, $v$. Having a separate update rule for the stop phase, where the avatar velocity exponentially decays to 0 , helps avoid sliding of the avatar if the user abruptly stops after lifting or pressing their foot, as demonstrated in Figure 5. Design coefficients, $c_{1}$ and $c_{2}$, define how fast the current values of the modelled and the avatar's velocities are updated. In this sense, velocity update expressions (1) and (3) represent first-order low-pass filters, where coefficients $c_{1}$ and $c_{2}$ can be computed based on required cut-off frequencies. In this paper, the coefficients were set to $c_{1}=0.02 / \Delta t$ and $c_{2}=0.1$ for cut-off frequencies $50 \mathrm{~Hz}$ and $10 \mathrm{~Hz}$, respectively. The cut-off frequencies define the VR avatar movement dynamics and can be adjusted to achieve the required behaviour. The avatar velocity calculation algorithm is also robust against stop regions that do not appear at the maxima points of platform movement. These points are called intra-step stops and are neglected from velocity estimation. Table 2 summarises the parameters and variables of the proposed walking velocity calculation method. The proposed algorithm provides natural walking generation for a VR avatar control interface, but to achieve this a user is required to perform consequent left/right foot stepping actions. It was demonstrated in our experiments, that such gestures are easy to learn and implement with our ankle actuation platform.

\subsection{Demonstrating the walking algorithm}

In this section, we present a VR locomotion mapping example with three different gesture input frequencies taken from the same user to provide a better understanding of how the algorithm works. The gesture input and avatar movement data, shown in Figure 5, were recorded in the video-based walking movement replication tests (described in section 4.1) where a participant replicated physical walking (demonstrated in the video at walking speeds 5, 6, and $7 \mathrm{~km} / \mathrm{h}$ ) with foot stepping gestures using the ankle platform. The first plot in Figure 5 presents the user's ankle input (angle) for three different walking speeds. The last two plots in Figure 5 show the calculated avatar walking speed 
a) Gesture input: ankle platform angle

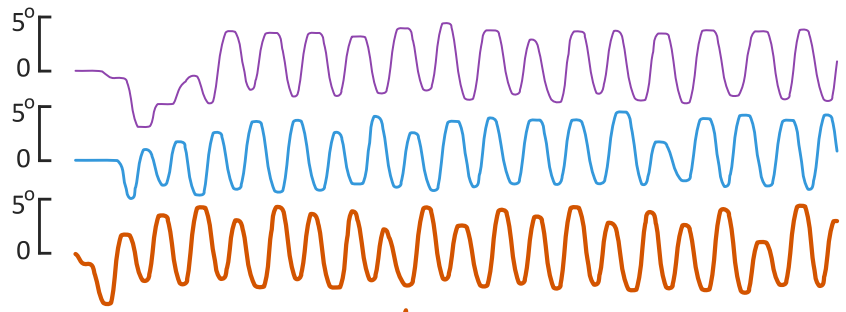

b) Avatar speed, $\mathrm{km} / \mathrm{h}$

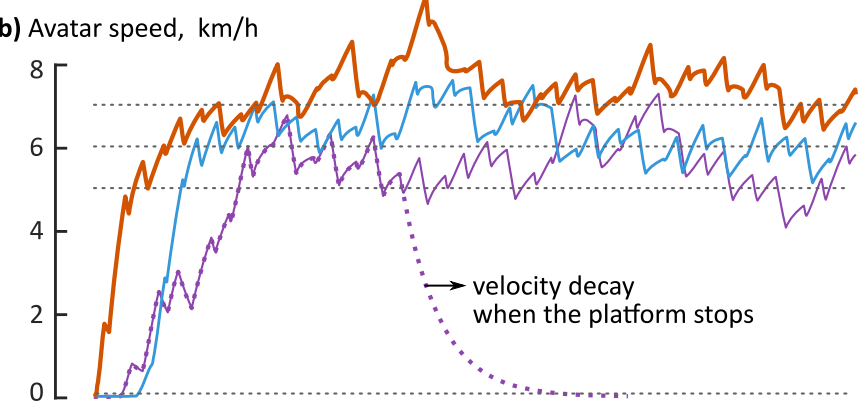

c) Avatar displacement, $\mathrm{m}$

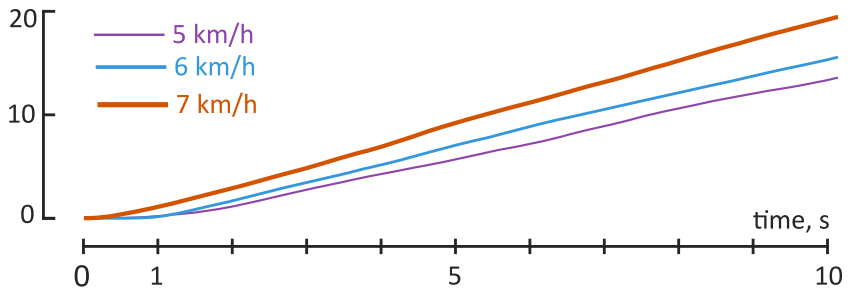

Fig. 5. Experimental results from a typical subject demonstrating, a) the ankle platform angle, b) generated avatar speed, and c) the avatar's displacement, recorded from a user mimicking physical walking. Physical walking was demonstrated with a video of a person walking on a treadmill at speeds 5,6 , and $7 \mathrm{~km} / \mathrm{h}$. The dashed line in the avatar speed plot demonstrates how the velocity would decay if the user suddenly stopped stepping. The same time axis is used for all plots.

and displacement in the VR scene. The resulting motion of the avatar corresponds well to the ankle movements. The user was able to perform regular stepping action and smooth displacement of the avatar was achieved through the integration of the calculated avatar speed. The speed of the avatar increased from 0 to a reference point when the user started foot stepping gestures (approx. $t=0.4 \mathrm{~s}$ ). Relatively small oscillations of the calculated speed were observed because of the switching nature of our algorithm (the avatar switched to decelerated movement at every stopping region in the gesture); however, this switching did not affect the resulting avatar displacement. As it was expected by the design, the intensity (frequency) of stepping motion directly influenced the walking speed of the avatar.

\subsection{Integrating steering strategy}

To transfer our walking method to a 3D scenario we need to have a method for turning around the longitudinal axis of the avatar.

We have implemented three techniques for the avatar turning control which are demonstrated in Figure 6 . All techniques employ the movement of the head mounted display to trigger steering during locomotion. The angular difference of the head or body orientation from neutral

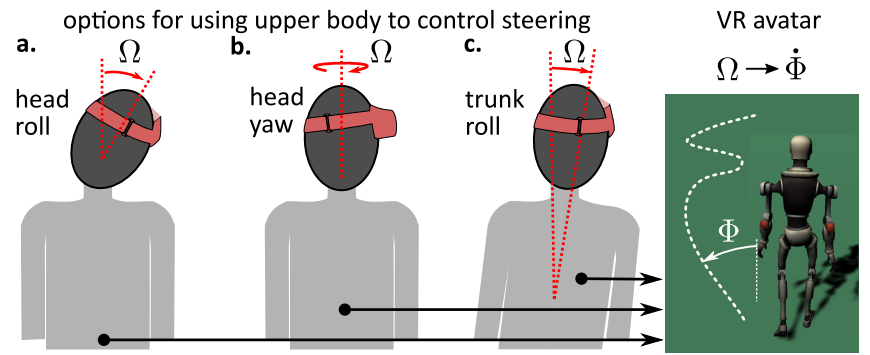

Fig. 6. Options to use upper body movements: a) head roll; b) head yaw c) body sideways bending, to control steering during locomotion in VR.

upright and straight orientation, denoted by $\Omega$ in the figure, is mapped to angular velocity of the avatar in the horizontal plane as described in (4).

$$
\begin{aligned}
& \dot{\Phi}=\mathrm{ST}(\mathrm{DZ}(\Omega)), \\
& \Phi=\Phi^{-}+\dot{\Phi} \Delta t_{u}
\end{aligned}
$$

where $\Phi$ and $\Phi^{-}$are current and previous headings of the avatar; ST $(\cdot)$ and $\mathrm{DZ}(\cdot)$ are saturation and dead-zone functions. Measured angular orientation of a user's head or upper body, $\Omega$, is initially subjected to the combination of dead-zone and saturation functions to avoid unintentional turning and protecting users from performing sudden unwanted turning movements. Parameters of these functions are currently tuned at a comfortable level for the authors, but these could be further studied to get a general view of their effect on intensifying motion sickness. Combining equations presented in this section, a VR avatar can be navigated inside a 3D environment.

The head roll movement is rarely used since it is mostly preferred for either looking under objects or matching a straight perspective with tilted objects. Therefore, it can be spared for avatar steering and would not cause much upper body movement. The head yawing motion may feel more intuitive for some but forbids the user from walking straight and gazing around simultaneously. Torso movement can provide the sense of body inclination to resemble real turning but requires reliable measurement of the headset's spatial position. We currently use head roll movement in our further studies. The biggest challenge in turning strategies is to understand which method is less prone to intensifying simulator sickness. However, the evaluation of turning algorithms is out of the scope of this study.

\section{HAPTIC TERRAIN FEEDBACK}

The ankle platform designed for intuitive walking in VR is equipped with an electric actuator enabling generating force feedback (in the form of programmable toques applied to feet and ankle joints) which can be used to render terrain or slope information. In this section, we demonstrate how different types of terrains can be rendered with our device. In particular, we describe three common terrain types that were used in terrain classification experiments. 

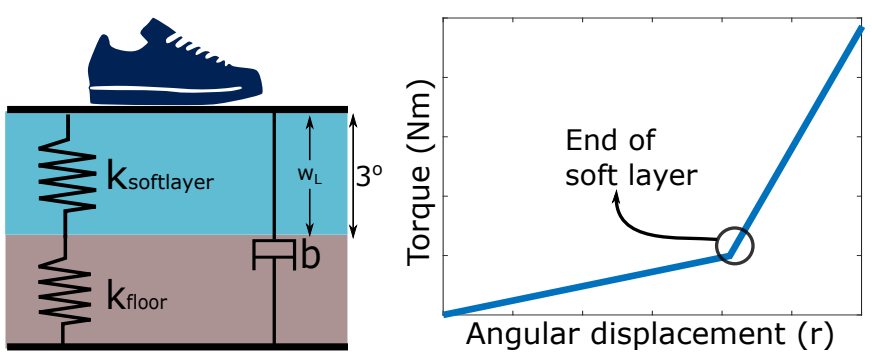

Fig. 7. Left: a depiction of a single layer possessing softer stiffness placed on the floor layer with the same stiffness as the stiff terrain. Right: highlights a transition from softer to higher resistance.

TABLE 3

Layer parameters

\begin{tabular}{l|l|l} 
Parameter & Explanation & Value \\
\hline \hline$k_{\text {floor }}$ & Ground stiffness & $1.5 \mathrm{Nm} / \mathrm{deg}$ \\
$k_{\text {soft }}$ & Soft layer stiffness & $0.3 \mathrm{Nm} / \mathrm{deg}$ \\
$n_{L}$ & Number of layers & 5 \\
$w_{L}$ & Layer width & $3 \mathrm{deg} / n_{L}$ \\
$S_{y}$ & Minimum yield strain of layer & $0.1 w_{L}$ \\
$S_{i n c}$ & Yield strain increment between layers & $0.04 w_{L}$ \\
$k_{b}$ & Stiffness of a brittle layer & calculated \\
$k_{b \max }$ & Maximum stiffness of brittle layers & $20 \mathrm{Nm} / \mathrm{deg}$ \\
$k_{\text {dec }}$ & Stiffness decrement between layers & $2 \mathrm{Nm} / \mathrm{deg}$ \\
$k_{b p f}$ & Stiffness post-failure & $2 k_{b}$
\end{tabular}

Stiff rigid terrain was modeled as a single stiff springand damper- based virtual floor, implemented in the impedance mode:

$$
\begin{aligned}
\Theta<0: \tau_{\text {ankle }} & =k \Theta+b \dot{\Theta} \\
\text { otherwise : } \tau_{\text {ankle }} & =0
\end{aligned}
$$

with $k$ and $b$ being stiffness and damping of the virtual floor surface. The parameters for this and other terrain models are shown in Table 3 .

Soft layered terrain was modelled as an addition on top of the stiff terrain. Soft terrain had a softer spring and the same damping element. The springs were not modelled to be serial, but the force was kept continuous by initiating the ground spring from a readily contracted state. After passing the soft layer region, the ground stiffness was applied as depicted in Figure 7

Brittle layered/crunchy terrain was modelled as a composition of several layers stacked on top of the stiff ground. Each layer included a stiffer spring compared to the ground, and the torque felt by the user was computed as the serial combination of all springs. The brittle layers, modelled like brittle materials which would undergo linear elastic deformation until they break after a certain compressing strain, aligned with Mohr-Coulomb yield criterion. Since successive cracking sensations were desired, the materials were designed to crack at different compressing stress and strain values. Once a layer was broken, it shrunken to around half of its original size, and the post-failure stiffness of the layer, $k_{b p f}$, is set as twice the initial stiffness, $k_{b}$, increasing the overall stiffness. Several previous works used vibration actuated floor tiles to generate a sensation of brittle terrain [34]. These works generally use force sensors under the stepping tile to understand the amount of force exerted by the user on the ground and use it in the dynamics calcu-
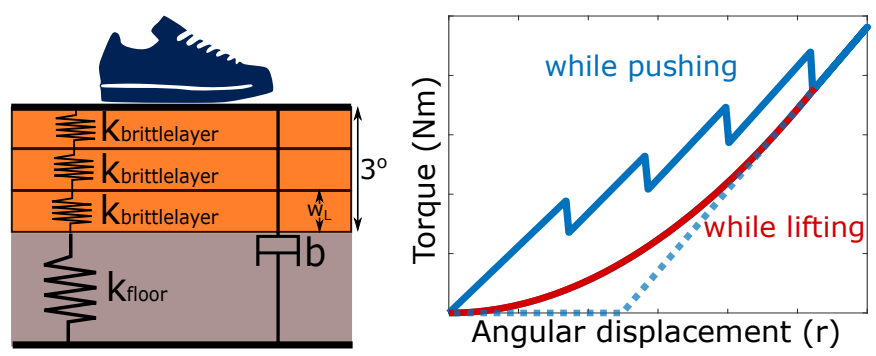

Fig. 8. Left: a depiction of several high stiffness, but low strength, layers placed on the floor layer with the same stiffness as the stiff terrain. Right: saw-tooth like force vs. displacement pattern occurring due to material failure on the right. The regularity of the pattern is due to the linear distribution of the stress-strain curves of separate layers and can be randomized. The blue and red lines present the force profiles applied while pushing down and lifting one's foot, respectively. The dotted line demonstrates the resulting force profile if the nonlinear spring model was not utilized during lifting.

lations of their Fiber Bundle Model. The fiber break events are used to calculate the vibration feedback. In our case, the deflection of the platform is used as the input displacement of layers. Our force kinesthetic feedback calculation scheme focuses on generating a breaking sensation that is kinesthetically more distinguishable. Users feel a temporary relaxation upon breaking a layer, and this is followed by a stiffer resistance. This is illustrated by saw-tooth shaped displacement vs. force relationship in Figure 8 . The red portion of the force signal in the figure shows the springapplied when the user finishes pushing down and starts to lift their foot. The reason for implementing a nonlinear spring instead of the force profile represented by the dotted line is to guide the platform to the above horizontal level.

\section{EXPERIMENT 1: LEARNING WALKING GESTURES}

\subsection{General conditions}

In this section, we present our first study, aimed at understanding if users can efficiently learn and use the interface to walk in VR. The experiments were conducted in accordance with guidelines specified in Queen Mary Policy on Research with Human Participants and was reviewed and approved by the Queen Mary University Ethics Committee.

Figure 1 shows the experimental setup used during the study. A participant sat comfortably on an adjustable chair with both feet placed on the actuated ankle platform. An Oculus Rift head-mounted VR display and a highperformance computer were used to recreate the VR scene. The VR scene was developed with the Unity 3D game engine. The developed robotic ankle platform was controlled in a custom-designed C\# application which communicated with the VR scene to read the participant's feet-based gestures for walking.

By default the ankle platform was controlled in impedance mode to maintain a horizontal position in the absence of user inputs; however, the platform was compliant, allowing a user to perform stepping gestures and tilt the platform. All experiments were conducted in a quiet laboratory environment. White noise was played through earphones during the trials to block any cognitive effects from acoustic noises created by the platform's actuator. 
All human participants were healthy. They all had prior experience with HMDs but did not use VR applications regularly. None of the participants had experience with walking interfaces for VR. Before the experiments, the participants were asked for their weight and height to adjust the seat position to an appropriate setting with respect to the ankle platform, such that they could comfortably apply forces on the platform. The weight of the user was also important as it changed the level of the force they used to apply to the platform. After each trial, the participants were asked if they required resting time or if they felt any dizziness. All participants were offered short breaks between the trials.

\subsection{Methods}

We investigated whether the proposed walking gesture was easy to learn for walking in VR. Eleven participants (ages 24-30, 2 females, heights $164-191 \mathrm{~cm}$, body mass $58-90 \mathrm{~kg}$ ) took part in Experiment 1: Learning Walking Gestures . The task was to perform VR locomotion through the movement of a virtual avatar along a straight path to reach the final point as seen in Figure 1 Only visual feedback through the HMD was provided to the participants. The small cubes suspended in the virtual scene were intended to aid the user's visual sense of movement without causing much distraction. The participants were asked to use the designed platform with the proposed walking gesture on three different days (sessions): first encounter with the device (Day 0), a day, approximately 24 hours, after the first trial $($ Day+1), and a week after the first encounter (Day+7). Each session included 10 trials. Each trial lasted approximately 1 minute. No training trials were organised in this study, as the goal was to observe learning of the proposed walking gestures. Users were instructed to walk at a speed that was comfortable for them without stopping until the end of the path. The stiffness of the platform was scaled with the weight of the participant. The virtual speed of the avatar, number of intra-step stops, and percentage of stop phase were recorded in each trial for each participant during the three sessions. Intra-step stop regions, defined in Section 3.2 refer to the intermediate stopping regions caused by simultaneous plantar flexion of one ankle and dorsiflexion of the other. The number of intra-step stops defines the number of mistaken gestures when human participants stepped with one foot while lifting the other. The percentage of stopping regions refer to the percentage of time taken during which the platform angular speed is below the threshold value. This value decreases as the user improves the movement rhythm and starts to move more fluently. One-way ANOVA and posthoc comparisons using the Tukey HSD test were used to analyse the statistical significance of the results.

Qualitative survey. At the end of all three sessions (after Day+7), the participants were asked to rate the proposed VR walking interface at a scale of 1 (very bad) to 10 (very good) on five factors listed below (the neutral response was equivalent to 5.5). The meaning of each factor was explained to users.

- Naturalness: How much does it resemble walking? Do you feel like your steps move you like your steps would in a real environment?

- Ease of use: How easy is it to get used to the interface?

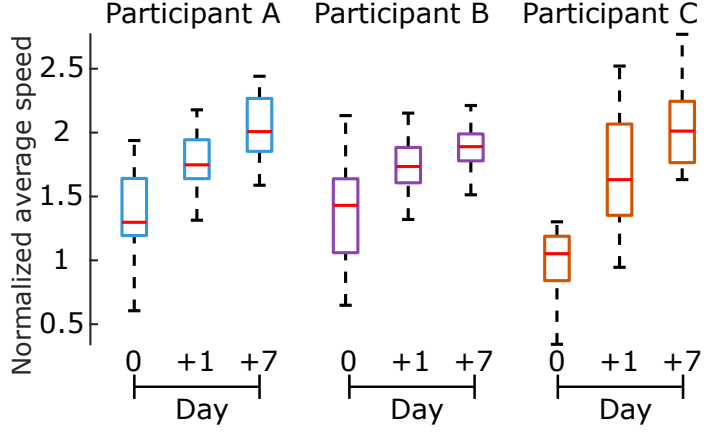

Fig. 9. Average VR walking speed, performed by three different randomly selected participants during each experimental session. The bottom and top limits of the boxes denote the first and third quartiles. The whiskers reach out to the last values that are in the range of two interquartile distances from the median. Similar results were observed in all other participants.

- Motion sickness: How much motion sickness, in other words, nausea or dizziness, does using the interface cause?

- Tiredness: How tired do you feel after using it?

- Comfort: Are you comfortable with the motion you are required to do for using the device?

We have further clarified to participants that comfort relates to the ease of performing the required posture and the feet movement, whereas tiredness relates to the overall exhaustion the task causes until completion. The naturalness of the stepping movement is one of the important contributors to immersion.

\subsection{Results}

During the learning walking gestures experiments, we recorded ankle platform angles and computed the speed of the VR avatar for each participant in each trial. These data were used to analyse the speed of virtual walking and the smoothness and robustness of participants' gesture inputs. All participants completed the walking task successfully. On average, across all participants, it took $40.4 \pm 4.36$ (mean \pm st.dev.) steps comprising left and right stepping gestures.

Walking speed. Figure 9 presents the normalized average speed of virtual walking for three typical participants for each of the three sessions (days). The reported speed of the avatar in each trial was calculated by measuring the time it took the avatar to travel from the starting point to the final point (indicated visually in the VR scene). The obtained speed values were normalized by dividing all the results by the mean value of the first trial of Day 0 of all participants. For all participants, it was observed that the normalized average speed of virtual walking increased from Day 0 to Day+7, demonstrating that it was easy to start using the interface and improve walking performance over time. These results were observed in all participants, and therefore in the following, we present the combined results across all participants.

Figure 10 presents the results across all participants during the learning walking gestures experiment for all 10 trials in each of the three sessions. Figure 10p shows the normalized average speed for all participants during each 
trial in three sessions. Figure 10 a shows that the virtual walking speed increased from Day 0 to Day +1 and Day +7 . More importantly, we can observe that the speed of walking increases notably in the first 10 trials in Day 0 . Sessions of Day +1 and Day+7 also demonstrate a slight increase in the average speed of walking across all participants; however, that was not statistically significant. It is clearly observed that the participants learnt the walking gestures during Day 0 and were able to repeat the trials and demonstrate improved performance one day, and one week later without losing the learnt walking gestures. Day by day comparisons of results show that there is a performance difference $(F(2,327)=73.69, p<0.001)$ on different days. Post-hoc comparisons using the Tukey HSD test indicated that the mean of average speed values in each day are significantly different from one another.

Gesture errors and smoothness of walking. Figure $10 \mathrm{p}$ presents the number of intra-step stops counted during each trial. It can be observed that the participants tended to have more asynchronous movements (mistaken gestures) in the earlier trials, and these diminish exponentially as the participants get more skilled. The last metric, shown in Figure $10 \mathrm{k}$, is the percentage of time spent in stop mode which would decrease as the users got more accustomed to the rhythmic stepping movements. Therefore, as demonstrated in Figure 10 $(b, c)$, decreasing the value of these metrics and of the number of intra-step stops indicates that the participants were improving their walking gestures. Day by day comparisons of results for the number of intra-steps stops and percentage of stopping regions show that there is a significant difference in user performance $(F(2,327)=24$, $p<10^{-} 9$ and $\mathrm{F}(2,327)=32.64, \mathrm{p}<0.001$ respectively) with respect to days. Post-hoc comparisons using the Tukey HSD test indicated that mean values attained for both metrics on Day 0 are significantly different than ones attained on Day +1 and Day +7 , but the mean results from Day+1 and Day+7 did not significantly differ.

To analyse the effect of having more trials in a day on learning the proposed walking gesture formally, we calculated the linear least squares regression on the data of Figure 10 for each of the three experimental sessions (days). The significance of the linear models was compared to the null hypothesis (having more practising trials does not affect the results) using a one-way ANOVA test. The slopes, $p, F$, and $R^{2}$ values of the resulting linear models are presented in Table 4 . The slope was negative for Day 0 and Day+1 due to the number of intra-step stops (failures) and percentage of time spent in stop mode, demonstrating that the VR walking performance was improving mainly during the first 10 trials in Day 0 and some improvement is also observed in Day+1 with a flatter slope. The VR
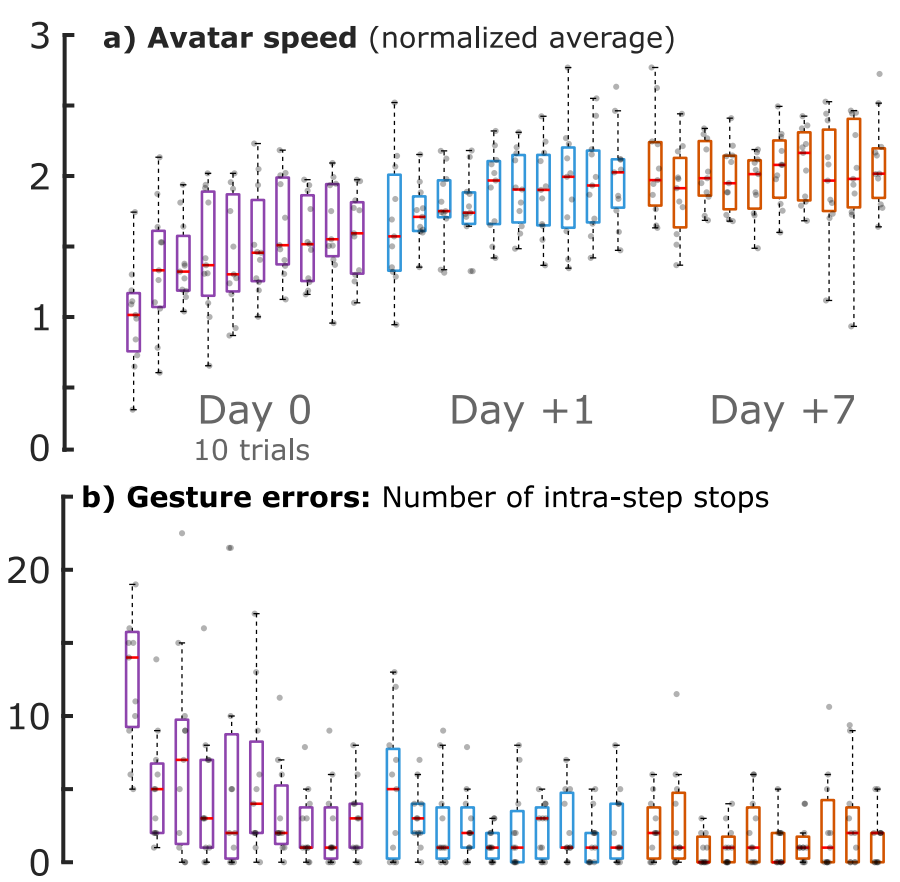

c) Gesture errors: Percentage of the stopping mode

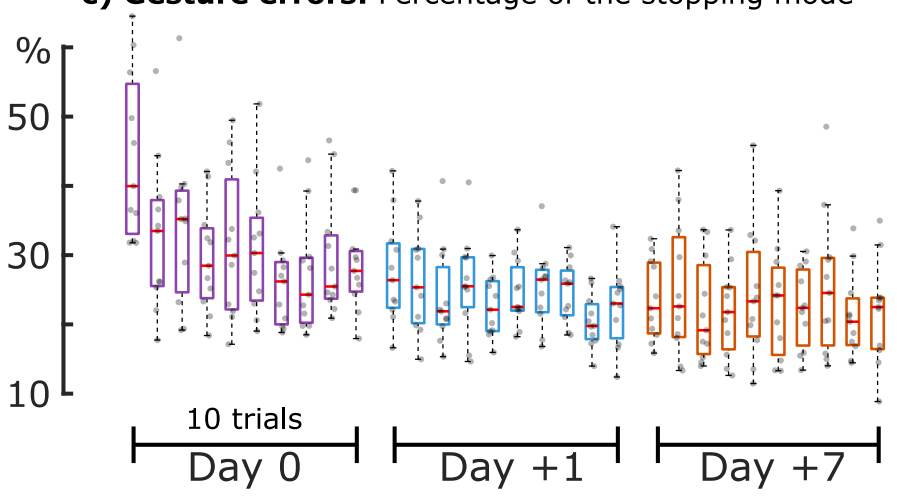

Fig. 10. Experimental results for learning walking in VR gestures recorded across all subjects for three sessions: a) avatar speed; b) number of intra-step stops; c) percentage of stopping mode. The lower, upper, and middle lines of boxes correspond to the 25th percentile, 75th percentile, and median, respectively. The whiskers at the top and bottom correspond to the minimum and maximum values.

walking speed was also increasing during the 10 trials of Day 0 , followed by gradually stabilising speed in Day +1 and Day+7. The calculated $F$ and $p$ values indicate that having more trials in Day 0 and Day +1 provide significant improvements in the average performance of the users. However, having more trials during the last session will not change the overall outcomes of the experiment which demonstrates that the participants' performance reached a certain level, and they can maintain the acquired motor skill.

TABLE 4

Linear regression analysis for learning walking gestures with ANOVA results

\begin{tabular}{c||ccc|ccc|ccc}
\multicolumn{1}{c||}{} & \multicolumn{3}{c}{ Average speed } & \multicolumn{3}{c}{ Number of failures } & \multicolumn{3}{c}{ \% of time in stop mode } \\
& Day 0 & Day+1 & Day+7 & Day 0 & Day+1 & Day+7 & Day 0 & Day+1 & Day+7 \\
\hline Slope & 0.16 & 0.11 & 0.021 & -0.90 & -0.33 & 0.20 & -1.17 & -0.55 & 0.02 \\
$R^{2}$-value & 0.15 & 0.09 & 0.004 & 0.19 & 0.04 & $10^{-4}$ & 0.13 & 0.06 & 0.003 \\
F-value & 18.85 & 10.99 & 0.44 & 7.02 & 3.47 & 1.25 & 10.03 & 6.03 & 0.01 \\
p-value & $<0.001$ & $<0.001$ & $>0.9$ & $<0.001$ & $<0.001$ & $>0.08$ & $<0.001$ & $<0.001$ & 1
\end{tabular}


The experiment demonstrated that all participants learnt the walking gesture during the first 10 trials and no relearning was required at later sessions, as they were able to retain this skill. The resulting low $R^{2}$-values show that linear models fitted to trial count do not necessarily explain a great percentage of the variation in the data. This may be due to factors such as non-linear learning curves, users not adjusting at the same rates or trials, or the effect of fatigue on some users. Although modelling the learning curve as a linear function is not ideal, it helped us understand the general improvement trends on each day and how much practice is required to use the device efficiently.

Usability survey. Figure 11 presents the results of the qualitative survey from 11 subjects (completed after the third session of the learning walking gestures experiment at Day+7). On a scale from 1 to 10 , the mean of reported ratings indicates that the proposed walking in place technique did not cause any simulator sickness $(\approx 9.6$ or $95 \%)$, it was easy to use $(\approx 7.6$ or $73 \%)$ and was not tiresome $(7.2$ or $69 \%)$. The naturalness of the walking motion, comfort were marked at $\approx 6.9$ or $65 \%$ and $\approx 7.3$ or $70 \%$, respectively. One sample t-test showed that all criteria were statistically favoured by the participants (reported higher than the neutral response of 5.5).

Several of the users considered the system considerably fatiguing after the experiments, however, none of the participants required a break between sessions (a break session was offered to all participants). The large variance and lower statistical significance in fatigue responses might be explained by the different heights and weights of some participants. We have used two strategies to mitigate the influence of users' height and weight on the task performance and improve the user experience. We made the chair height and distance adjustable to provide the same sitting posture for all participants and scaled the overall stiffness of the platform with the weight of the user before starting the experiments. Among all participants, seven reported that the interface does not cause much fatigue (not tiring factor more than 5.5), and four participants provided responses lower than 5.5. A two-sample t-test showed that users who are taller than $165 \mathrm{~cm}$ or heavier than $65 \mathrm{~kg}$ characterised the interface as not fatiguing $(p<0.001$ for taller and $\mathrm{p}<0.05$ for heavier participants, respectively). We may have adjusted the algorithm parameters or seat position to favor the users with medium and bigger body sizes. In future work, we will look further into the reasons behind fatigue and possible improvements through changes in system parameters.

\section{EXPERIMENT 2: TERRAIN CLASSIFICATION}

\subsection{Methods}

In this section, we present our second study, aimed at verifying if the proposed interface can be used to render haptic feedback associated with different types of virtual terrain. The same general experimental conditions (see 6.1) were used in this experiment. Nine participants (ages 2430, including 2 females; different participants from experiment 1) took part in Experiment 2 Terrain Classification. The participants were asked to use the ankle platform to walk along a predefined straight path in a VR scene. Three different virtual terrains were implemented: (1) stiff floor, (2)

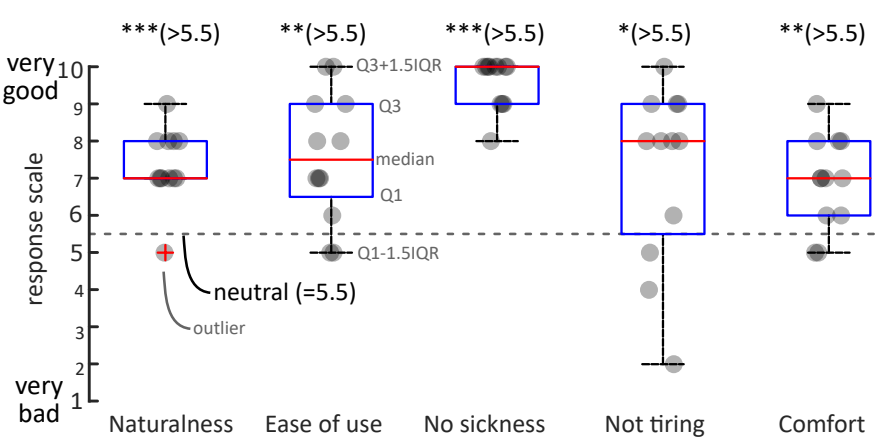

Fig. 11. Box plot of the qualitative survey results where the lower, upper, and middle (red) lines of boxes correspond to the 25th percentile, 75th percentile, and median, respectively. One-way t-test results are presented on the top of the boxplot corresponding to each column. The grey dashed line corresponds to the neutral score of 5.5 which is used for the t-test comparison.

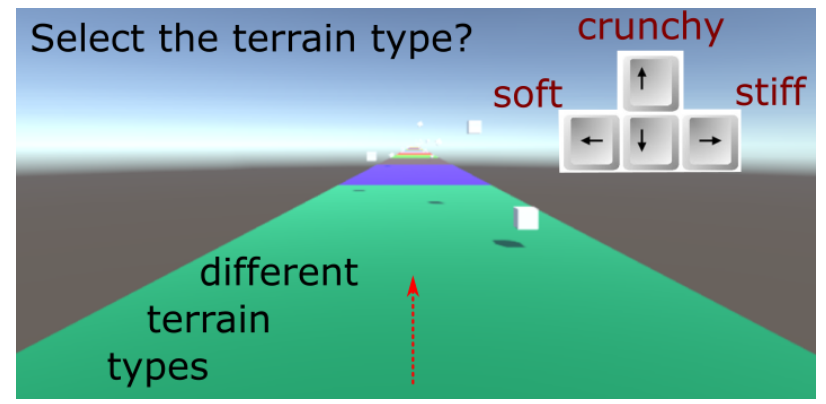

Fig. 12. VR scene for the terrain classification experiments.

stiff floor with a soft layer on top, (3) stiff floor with brittle layers on top (see Section 5 . The floor of the virtual path was divided into 18 sections, each randomly assigned with one of the three terrain types, such that each terrain type was included in each path six times, as seen in Figure 12 The sections were randomly coloured, such that the virtual floor colours did not correspond to any terrain type. Each participant had three trials in this experiment, and each trial had different random arrangements of the terrain types. The subjects were informed that they would be walking on three different terrain types and what these types of terrain would be. The participants were instructed to identify and report the terrain type at the end of each visually marked terrain section. A computer keyboard (three cursor buttons) was used to record their answers. The participants were allowed to stop to think about their decision but they would not get additional information about the terrain without stepping. The number of correctly classified terrains were counted for each participant.

\subsection{Results}

All participants were able to correctly identify the virtual terrain types in at least $70 \%$ of cases. Figure 13 shows the classification results in the form of a confusion matrix with additional data on accuracy (ratio of correctly predicted observation to the total observations), precision (ratio of correctly predicted positive observations to the total predicted positive observations), recall (ratio of correctly predicted positive observations to all observations in actual class), and F1 scores (the weighted average of precision and 


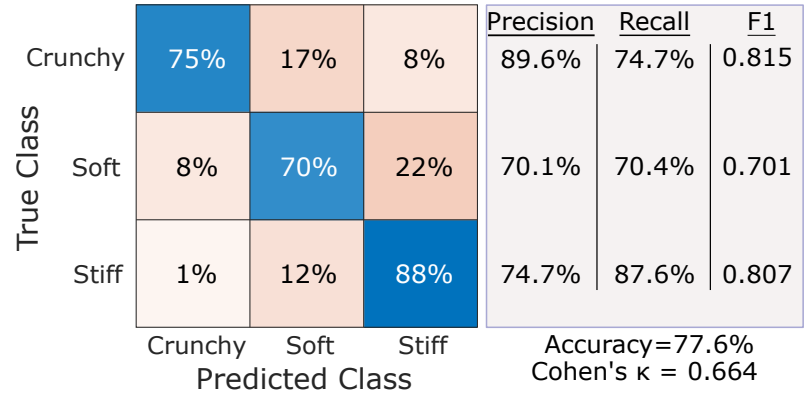

Fig. 13. Results for haptic terrain classification accuracy (\% of answers).

recall) for each terrain type. The average correct response rate of each individual participant varied between $65 \%$ and $95 \%$. The precision and recall rates for all terrains were higher than $70 \%$. Crunchy terrain had the highest precision, meaning that when users thought a terrain was crunchy, they were approximately $90 \%$ right. Users could identify the stiff terrain approximately $88 \%$ of the time the terrain was actually stiff. The F1 scores of both stiff and crunchy layered terrains are higher than 0.8 , and soft terrain is approximately 0.7 . This shows that the soft terrain was not as distinguishable as the other terrains. The overall terrain identification accuracy of the users was approximately $78 \%$. The Cohen's $\kappa$ coefficient (which uses observed and expected accuracy values to rate a classifier between -1 and 1) was calculated as 0.664 , indicating that the observed terrain classification results are substantially reliable $(\kappa$ values above 0.6 are considered substantial [35], [36]).

There was confusion in identifying crunchy terrain as soft $(17 \%)$ and soft terrain as stiff $(22 \%)$. We believe that the main reason for such misclassifications was that our terrain rendering model did not perform as expected at higher angular velocities. In the case of fast ankle dorsiflexion, some users may not perceive smaller changes of torque feedback (like in the saw-tooth like pattern, Figure 8p) causing confusion between soft and crunchy terrains. That could also cause the users not to perceive the soft region of a soft terrain and assume that they have directly encountered the stiff region. The terrain classification experiment required users to distinguish different ground impedance characteristics rather than specific terrains. We will continue to work on the generation of specific terrain sensations using described impedance characteristics. Very few results are available for the classification of terrains through footbased haptic feedback devices. A multisensory terrain classification study performed in [37 showed that using only kinesthetic feedback is more challenging than using other modalities, such as vibrotactile feedback. We believe that our results are promising, and the feedback quality of the interface can be further improved and combined with other modalities for a more immersive experience in the future.

\section{Conclusion}

We developed and experimentally evaluated a novel interaction technique for walking in VR. Our method combines an integrated novel walking-in-place technique and haptic feedback to display different types of virtual terrains during walking. The interaction methods were implemented on a custom-designed low-cost, compact, and easy-to-use 1DoF ankle platform capable of simultaneously acting as an input device for an intuitive walking interface and as an output haptic feedback device to provide terrain information. Experimental studies demonstrated that the proposed interface can be used efficiently for interactive walking in VR and has significant potential in the haptic rendering of different types of virtual terrains. In contrast to previous works, our system uses only one actuator and angular position sensor, making the platform cost-efficient and easy to program. The proposed interface is designed to be used by seated users; therefore, it not only enables the use of wearable and handheld interfaces but also desktop control interfaces.

The limitations of the presented study will be addressed in future work, as the primary goal of this paper was to present the novel interface and demonstrate its key functions. It would be insightful to compare our system with existing seated and standing gesture inputs for locomotion in VR. Holding another qualitative user evaluation at a comparative study would provide more cogent results, as participants would have a clearer reference for comparison of parameters. Similar to the majority of previous works, our movement mapping algorithm generates forward body speed and does not simulate exact movements of the legs. To address this limitation, we are working on an improved version of the presented algorithm that calculates the phase of the gait cycle and step length to allow using legged avatar models and their walking simulations to improve the sense of body ownership [38]. Currently, the device does not sense which foot the user is stepping. The presented algorithm does not animate the avatar's legs, but for a whole-body animation algorithm, we may need to add additional contact sensors to sense if either foot is lifted or pressing on the platform. Furthermore, a comparison of steering techniques during locomotion with the presented interface is required, as it is essential to avoiding motion sickness. Likewise, we will explore methods, like stepping while leaning backwards, for walking backwards. We will also evaluate the cognitive load of the locomotion task to understand its effect on the performance of a simultaneous manipulation task. The above issues will be addressed with other types of VR motion interfaces being developed at our group [39], [40].

\section{ACKNOWLEDGMENT}

Authors would like to thank A. Smiles for proofreading the manuscript and A. Birn-Jeffery and S. C. Miller for fruitful discussions. This work was supported by the UK EPSRC grant EP/R02572X/1 National Centre for Nuclear Robotics.

\section{REFERENCES}

[1] R. A. Ruddle and S. Lessels, "For efficient navigational search, humans require full physical movement, but not a rich visual scene," Psychol. Sci., vol. 17, no. 6, pp. 460-465, 2006.

[2] M. Slater and M. Usoh, "Body centred interaction in immersive virtual environments," in Artificial Life and Virtual Reality, 1994, pp. $125-148$.

[3] M. Usoh, K. Arthur, M. C. Whitton, R. Bastos, A. Steed, M. Slater and F. P. Brooks, "Walking > walking-in-place $>$ flying, in virtual environments," in Proc, of the 26th Annual Conference on Computer Graphics and Interactive Techniques. USA: ACM, 1999, p. 359-364. 
[4] S. Razzaque, Z. Kohn, and M. C. Whitton, "Redirected Walking," in Eurographics 2001 - Short Presentations, 2001.

[5] R. P. Darken, W. R. Cockayne, and D. Carmein, "The omnidirectional treadmill: A locomotion device for virtual worlds," in Proc. of the 10th Annual ACM Symp. on User Interface Software and Technology, NY, USA, 1997, pp. 213-221.

[6] M. Slater, M. Usoh, and A. Steed, "Taking steps: The influence of a walking technique on presence in virtual reality," ACM Trans. Comput.-Hum. Interact., vol. 2, pp. 201-219, 1995.

[7] J. Feasel, M. C. Whitton, and J. D. Wendt, "LLCM-WIP: Lowlatency, continuous-motion walking-in-place," in 2008 IEEE Symposium on 3D User Interfaces, March 2008, pp. 97-104.

[8] J. D. Wendt, M. C. Whitton, and F. P. J. Brooks, "GUD-WIP: Gait-understanding-driven walking-in-place," IEEE Virtual Reality Conference, vol. 2010, pp. 51-58, Mar 2010.

[9] Y. Visell, J. R. Cooperstock, B. L. Giordano, K. Franinovic, A. Law, S. McAdams, K. Jathal, and F. Fontana, "A vibrotactile device for display of virtual ground materials in walking," in Haptics: Perception, Devices and Scenarios, M. Ferre, Ed. Berlin, Heidelberg: Springer Berlin Heidelberg, 2008, pp. 420-426.

[10] H. Son, H. Gil, S. Byeon, S.-Y. Kim, and J. R. Kim, "Realwalk: Feeling ground surfaces while walking in virtual reality," in Extended Abstracts of the CHI Conference on Human Factors in Computing Systems. ACM, 2018, pp. D400:1-D400:4.

[11] L. Bouguila, B. Hirsbrunner, M. Sato, and M. Iwashita, "Virtual locomotion interface with ground surface simulation," in ICAT, 2003.

[12] N. C. Nilsson, S. Serafin, and R. Nordahl, "The perceived naturalness of virtual locomotion methods devoid of explicit leg movements," in Proceedings of Motion on Games, ser. MIG '13. New York, NY, USA: ACM, 2013, pp. 133:155-133:164.

[13] L. Terziman, M. Marchal, M. Emily, F. Multon, B. Arnaldi, and A. Lécuyer, "Shake-your-head: revisiting walking-in-place for desktop virtual reality," in VRST, 2010.

[14] CyberShoes. [Online]. Available: https://www.cybershoes.io/

[15] J. N. Templeman, L. E. Sibert, R. C. Page, and P. S. Denbrook, "Pointman - a new control for simulating tactical infantry movements," in IEEE Virtual Reality Conference, March 2007, pp. 285-286.

[16] L. Bouguila, E. Florian, M. Courant, and B. Hirsbrunner, "Active walking interface for human-scale virtual environment," 2005.

[17] B. Williams, S. Bailey, G. Narasimham, M. Li, and B. Bodenheimer, "Evaluation of walking in place on a wii balance board to explore a virtual environment," ACM Trans. Appl. Percept., vol. 8, no. 3, pp. 19:1-19:14, Aug. 2011.

[18] S. Tregillus and E. Folmer, "VR-STEP: Walking-in-place using inertial sensing for hands free navigation in mobile vr environments," in CHI, 2016.

[19] J. Lee, S. C. Ahn, and J.-I. Hwang, "A walking-in-place method for virtual reality using position and orientation tracking," Sensors, vol. 18, no. 9, p. 2832, Aug 2018.

[20] R. P. Jayakumar, S. K. Mishra, J. F. Dannenhoffer, and A. M. Okamura, "Haptic footstep display," in 2012 IEEE Haptics Symposium (HAPTICS). IEEE, 2012, pp. 425-430.

[21] L. Terziman, M. Marchal, F. Multon, B. Arnaldi, and A. Lecuyer, "The king-kong effects: Improving sensation of walking in vr with visual and tactile vibrations at each step," in 2012 IEEE Symposium on 3D User Interfaces (3DUI), March 2012, pp. 19-26.

[22] G. Kato, Y. Kuroda, K. Kiyokawa, and H. Takemura, "Force rendering and its evaluation of a friction-based walking sensation display for a seated user," IEEE Transactions on Visualization and Computer Graphics, vol. 24, no. 4, pp. 1506-1514, 2018.

[23] S. Papetti, F. Fontana, M. Civolani, A. Berrezag, and V. Hayward, "Audio-tactile display of ground properties using interactive shoes," in Haptic and Audio Interaction Design. Springer, 2010, pp. 117-128.

[24] T. Yokota, M. Ohtake, Y. Nishimura, T. Yui, R. Uchikura, and T. Hashida, "Snow walking: Motion-limiting device that reproduces the experience of walking in deep snow," in Proceedings of the 6th Augmented Human International Conference, ser. AH '15. New York, NY, USA: ACM, 2015, pp. 45-48.

[25] G. Cirio, M. Marchal, A. Lécuyer, and J. R. Cooperstock, "Vibrotactile rendering of splashing fluids," IEEE Transactions on Haptics, vol. 6, no. 1, pp. 117-122, First 2013.

[26] R. Hidayah, S. Chamarthy, A. Shah, M. Fitzgerald-Maguire, and S. K. Agrawal, "Walking with augmented reality: A preliminary assessment of visual feedback with a cable-driven active leg exoskeleton (c-alex)," IEEE Robotics and Automation Letters, vol. 4, no. 4, pp. 3948-3954, 2019.

[27] C. T. Pan, Z. C. Lin, P. Y. Sun, C. C. Chang, S. Y. Wang, C. K. Yen, and Y. S. Yang, "Design of virtual reality systems integrated with the lower-limb exoskeleton for rehabilitation purpose," in IEEE International Conf. on Applied System Invention, 2018, pp. 498-501.

[28] H. Iwata, H. Yano, and F. Nakaizumi, "Gait master: a versatile locomotion interface for uneven virtual terrain," in Proceedings IEEE Virtual Reality 2001, March 2001, pp. 131-137.

[29] M. R. Chester, M. J. Rys, and S. A. Konz, "Leg swelling, comfort and fatigue when sitting, standing, and sit/standing," International Journal of Industrial Ergonomics, vol. 29, no. 5, pp. 289 - 296, 2002.

[30] B. B. Gibbs, R. J. Kowalsky, S. J. Perdomo, M. Grier, and J. M. Jakicic, "Energy expenditure of deskwork when sitting, standing or alternating positions," Occupational Medicine, vol. 67, no. 2, pp. 121-127, 082016.

[31] C. G. Horlings, M. G. Carpenter, U. M. Küng, F. Honegger, B. Wiederhold, and J. H. Allum, "Influence of virtual reality on postural stability during movements of quiet stance," Neuroscience Letters, vol. 451, no. 3, pp. 227-231, 2009.

[32] M. C. Whitton and T. C. Peck, Stepping-Driven Locomotion Interfaces. New York, NY: Springer New York, 2013, pp. 241-262.

[33] A. Otaran and I. Farkhatdinov, "Modeling and control of ankle actuation platform for human-robot interaction," in Towards Autonomous Robotic Systems. Springer, 2019, pp. 338-348.

[34] Y. Visell and J. R. Cooperstock, "Design of a vibrotactile display via a rigid surface," in IEEE Haptics Symposium, 2010, pp. 133-140.

[35] J. R. Landis and G. G. Koch, "The measurement of observer agreement for categorical data," Biometrics, vol. 33, no. 1, pp. 159174,1977

[36] M. L. McHugh, "Interrater reliability: the kappa statistic," Biochemia medica, vol. 22, no. 3, pp. 276-282, 2012.

[37] B. L. Giordano, Y. Visell, H.-Y. Yao, V. Hayward, J. R. Cooperstock, and S. McAdams, "Identification of walked-upon materials in auditory, kinesthetic, haptic, and audio-haptic conditions," J. Acoust. Soc. Am., vol. 131, no. 5, pp. 4002-4012, May 2012.

[38] M. Gonzalez-Franco, D. Perez-Marcos, B. Spanlang, and M. Slater, "The contribution of real-time mirror reflections of motor actions on virtual body ownership in an immersive virtual environment," 04 2010, pp. 111 - 114.

[39] A. Otaran and I. Farkhatdinov, "A cable-driven walking interface with haptic feedback for seated vr," in World Haptics Conference. IEEE, 2021, pp. 592-592.

[40] F. Soave, I. Farkhatdinov, and N. Bryan-Kinns, "Multisensory teleportation in virtual reality applications," in Conference on Virtual Reality and 3D User Interfaces Abstracts and Workshops. IEEE, 2021, pp. 377-379.

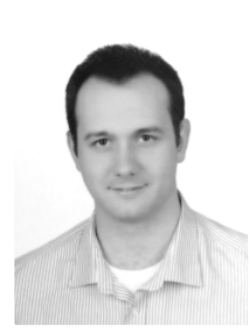

Ata Otaran is a Ph.D. Student in Robotics at the School of Electrical Engineering and Computer Science at Queen Mary University of London (QMUL). He got his M.Sc. in Mechatronics Engineering and B.Sc. in Mechatronics Engineering with minor in Mathematics from Sabanci University, Istanbul. His primary research interests are in the field of physical human-machine interaction, particularly use of haptics for virtual reality and educational interfaces.

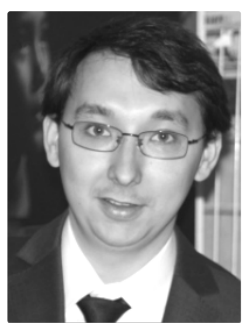

Ildar Farkhatdinov is a Senior Lecturer (Associate Professor) in Robotics at Queen Mary University of London and an honorary lecturer in the Department of Bioengineering of Imperial College of Science, Technology and Medicine, London, UK. He earned Ph.D. in Robotics in 2013 (Sorbonne University, France), M.Sc. in Mechanical Engineering in 2008 (KoreaTech, S. Korea) and B.Sc. in Automation and Control in 2006 (Moscow University STANKIN, Russia). 